\title{
SUR LA FRONTIÈRE DE MARTIN DES DOMAINES DE DENJOY
}

\author{
Alano Ancona
}

On considère dans ce travail la classe des domaines $\Omega$ de la forme $\Omega=\hat{\mathbf{C}} \backslash F$, où $\hat{\mathbf{C}}$ désigne la sphère de Riemann et $\mathrm{F}$ une partie compacte du graphe $\Gamma=$ $\{z=x+i y ; y=f(x)\}$ d'une fonction lipschitzienne $f: \mathbf{R} \rightarrow \mathbf{R}$. On dit que $\Omega$ est un domaine de Denjoy Lipschitzien, les domaines de Denjoy "classiques" correspondant au cas $\Gamma=\mathbf{R}$. Dans l'étude de la théorie des fonctions dans les ouverts peu réguliers, ces domaines constituent un champ d'investigation naturel ([B], [Ca], [G-J], [Z], [A1], [A2]).

On s'intéressera ici à la frontière de Martin ([Ma], [Na]) d'un tel domaine. Si $\Omega$ est de Green (ce qui équivaut à $F$ non polaire) et si $0 \in F$, on sait que le cône $C=\{u ; u$ harmonique $>0$ sur $\Omega \backslash F\} ; h=0$ sur $F \backslash\{0\}$ est de dimension 1 ou 2 , et qu'il est engendré par une ou deux fonctions harmoniques minimales sur $\Omega$. (cf. [A2]; [B], [A1] pour le cas où $F \subset \mathbf{R}$ ).

Lorsque $\operatorname{dim}(C)=1$, la convergence ordinaire d'un point variable de $\Omega$ vers 0 équivaut à la convergence de ce point, dans le compactifié de Martin de $\Omega$, vers le point minimal associé à 0 . On dira que 0 est un point Martin simple pour $\Omega$.

Lorsque $\operatorname{dim}(C)=2, C$ est associé à deux points minimaux du compactifié de Martin $\hat{\Omega}$ de $\Omega$; ces points sont les seules valeurs d'adhérence possibles sur la frontière de Martin minimale de $\Omega$ des suites de points de $\Omega$ tendant vers 0 au sens ordinaire. On dira alors que 0 est un point Martin double pour $\Omega$. $\mathrm{N}$. Chevallier [Ch] a précisé ces propriétés en montrant qu'on obtient les deux minimales $h$ et $k$ associées à 0 , en posant: $h(x)=\lim _{t>0, t \rightarrow 0} G(i t, x) / G\left(i t, P_{0}\right)$, et $k(x)=\lim _{t<0, t \rightarrow 0} G(i t, x) / G\left(i t, P_{0}\right.$ ) (en notant $P_{0}$ le point de normalisation choisi et $G$ la fonction de Green de $\Omega$ ). On sait aussi que $\lim _{t>0, t \rightarrow 0} h(i t) / k(i t)=\infty$.

L'article de Benedicks [B] fournit, lorsque $F \subset \mathbf{R}$, une caractérisation du cas où 0 est simple par la divergence d'une intégrale de certaines mesures harmoniques. Du critère de Benedicks découle en particulier que la dimension du cône $C$ est une fonction croissante de $F$, si $F$ est un fermé variable de $\mathbf{R}$. On peut aussi prouver cette propriété en la rattachant à la suivante: si $F \subset \mathbf{R}$, (ou plus généralement si $f$ est de classe $C^{1, \alpha}, 0<\alpha \leq 1$ ), et si $0 \in F$ est un point Martin double pour $\Omega=\hat{\mathbf{C}} \backslash F$, l'ensemble $\Gamma \cap \Omega$ est effilé minimal relativement à chacune des deux minimales associées à 0 (voir $[\mathrm{Ch}]$ ). 
Les problèmes suivants se posent alors naturellement [Ch]: si $\Gamma$ est une courbe lipschitzienne et $F$ un compact variable contenu dans $\Gamma$, la dimension de $C$ estelle fonction croissante de $F$ ? Dans le cas où 0 est un point double pour $\Omega, \Gamma \cap \Omega$ est-il effilé (minimal) en chacun des points minimaux associés à 0 ?

L'objet de ce travail est de montrer que ces questions admettent une réponse négative. On s'appuyera sur une étude préliminaire (qu'on espère intéressante par elle même), de certains domaines de Denjoy (en particulier ceux qui sont symétriques par rapport à l'origine), domaines pour lesquels on établira un critère d'effilement minimal (relativement à l'une des minimales attachées à 0 ) et un critère de simplicité pour 0 .

\section{Rappels et estimées préliminaires}

A. Rappelons d'abord une inégalité, essentielle pour la suite, du type "Harnack au bord" et relative aux domaines de Denjoy lipschitziens.

Soient $f:[-1,1] \rightarrow \mathbf{R}$ une fonction $K$-lipschitzienne $(K>0)$, telle que $f(0)=0$ et $F$ une partie fermée du graphe $\Gamma$ de $f$.

Théorème 1.2 ([A.2]). Soient $u, v, w$ trois fonctions harmoniques $>0$ sur le domaine $V=U \backslash F, U=\{x+i y ;|x|<1,|y|<4 K\}$, u et $v$ s'annulant sur $F$, et soient $A=2 K i, B=-2 K i$. On a alors, pour $z \in\left\{\frac{1}{2} \partial U\right\} \cap V$,

$$
u(z) \leq c\{[u(A) / v(A)] v(z)+[u(B) / w(B)] w(z)\}
$$

pour une constante $c>0$ ne dépendant que de $K$.

Une forme équivalente de ce théorème est donnée par l'énoncé suivant. Soit $\omega$ un domaine greenien plan tel que $\omega \cap U=V$ et soit $\mathrm{u}$ une fonction harmonique $>0$ sur $V$ s'annulant sur $F$. On a alors l'estimée

$$
u(z) \leq c\left\{[u(A) / g(A)] g(z)+\left[u(B) / g^{\prime}(B)\right] g^{\prime}(z)\right\}
$$

pour tout $z \in \frac{1}{2} U$, où $g$ (respectivement $g^{\prime}$ ) désigne la fonction de Green de pôle $\frac{3}{2} A$ (respectivement $\frac{3}{2} B$ ) dans $\omega$.

Remarque 1.2. Le théorème 1.2 contient le principe de Harnack au bord standard pour les domaines de Lipschitz: Si $u, v$ sont deux fonctions harmoniques $>0$ sur $\omega=\{x+i y ;|x|<1, f(x)<y<4 K\}, u$ et $v$ s'annulant sur le graphe de $f$, on a $u(z) / u(A) \leq c v(z) / v(A)$, pour chaque $z=x+i y,|x| \leq 1, f(x)<y<2 K$ $(c>0, c(K)>0)$.

B. On dira que le domaine de Denjoy $\Omega=\hat{\mathbf{C}} \backslash F$ ( $F$ comme ci-dessus) est de type (B) à l'origine s'il existe $C>0$ tel que (on note $A_{t}=i t$ ) pour tout $s, t>0$

$$
G\left(A_{t},-A_{s}\right) \leq C G\left(A_{t}, A_{s}\right), \quad \text { et } \quad G\left(-A_{t}, A_{s}\right) \leq C G\left(-A_{t},-A_{s}\right) .
$$


(Il suffit de vérifier ces inégalités pour $s, t \leq 1$.) Si par exemple $F \subset \mathbf{R}, \Omega$ est de type (B). Plus généralement, s'il existe un disque ouvert $D$, de centre $A=i \varepsilon$, $\varepsilon>0$, et rayon $r=\varepsilon$, tel que $F \subset \mathbf{C} \backslash\{D \cup-D\}$, est de type (B), d'après [A1]. Il suffit même que $|f(x)| \leq C|x|^{1+\alpha}$, pour un $\alpha>0$, comme le montrent des modifications convenables de [A1]. Le point de départ ici sera l'observation suivante.

Proposition 1.3. Si $F$ est symétrique autour de 0 , alors $\Omega$ est de type (B).

Preuve. Soient $X=i t, t>0$ et $u(x)=G(X, x)$. Il est clair qu'il existe $C_{1} \geq 1$ tel que pour $Y=i t / 2$, on ait: $G(X,-Y) \leq C G(X, Y)$. S'il existe $t^{\prime}$, $0<t^{\prime}<\frac{1}{2} t$, tel que $G\left(X,-i t^{\prime}\right) \geq 2 C_{1} G\left(X, \frac{1}{2} i t^{\prime}\right)$ considérons le point $Z=i t^{\prime}$ correspondant à la valeur $t^{\prime}$ maximale. On a alors, d'après le Théorème 1.1 et l'égalité $G(X,-Z)=2 C_{1} G(X, Z)$,

$$
G(X, x) \leq C\left\{2 C_{1}+\left(2 C_{1}\right)^{-1}\right\} G(X,-x)=C_{2} G(X,-x)
$$

pour tout $x \in B\left(0, t^{\prime}\right)$. A fortiori, $G(X,-i s) \leq C_{2} G(X, i s)$ pour $0<s<t^{\prime}$. D'où l'énoncé. $\square$

C. Il sera commode de considérer la propriété d'uniforme régularité suivante (variante de celle de [A4]):

(UR) $F \neq \emptyset$ et il existe $c_{1}>0$ tel que pour tout $x \in F$ et $0<r \leq 1$, la mesure harmonique de $\partial \Omega \cap B\left(x, \frac{1}{4} r\right)$ dans $B(x, r)$ est minorée par $c_{1}$ sur $\partial B\left(x, \frac{1}{2} r\right)$.

On sait qu'alors la fonction de Green $G$ de $\Omega$ vérifie: $c^{-1} \leq G(x, y) \leq c$ pour $x \in \Omega, d(x, y)=\frac{1}{2} \delta(x), \delta(x)=d(x, \partial \Omega) \leq 1, c>0$ ne dépendant que de $c_{1}$. Notons d'abord une conséquence élémentaire et fort utile de l'uniforme régularité.

Proposition 1.4. Pour $x, y, z \in \Omega$ tels que $\delta(y)=d(y, F) \leq 1, x, z \notin$ $B\left(y, \frac{1}{2} \delta(y)\right)$, on a avec une constante $c>0$ ne dépendant que de $c_{1}: G(x, z) \geq$ $c G(x, y) G(y, z)$.

Il suffit d'observer que $u=G(x, \cdot)$ est de l'ordre de $G(x, y)$ sur $\partial B\left(y, \frac{1}{2} \delta(y)\right)$ (Harnack), et donc que $G(y, \xi) \leq c\{G(x, y)\}^{-1} u(\xi)$, si $\xi \in \partial B\left(y, \frac{1}{2} \delta(y)\right)$. Par le principe du maximum, cette inégalité est valable pour tout $\xi \in \Omega \backslash B\left(y, \frac{1}{2} \delta(y)\right)$, et au point $z$ en particulier. $\square$

Voici encore deux conséquences de (UR).

1.5. Il existe $\alpha \in] 0,1\left[\right.$ et $c>0$ tels que $G\left(A_{t}, A_{s}\right) \leq c(t / s)^{\alpha}$, si $0<2 t<s$ $\leq 1,\left(\alpha\right.$ et $c$ ne dépendant que de $c_{1}$ (dans UR) et de $\left.K\right)$.

1.6. $\omega \cap B(0,2 K+1)$ étant uniformément régulier au sens de [A4], il découle de $\left[\mathrm{A} 4\right.$, Théorème 1] qu'on a l'inégalité pour chaque $\varphi \in C_{0}^{\infty}(\Omega)$ :

$$
\int_{\delta(x) \leq 1} \delta(x)^{-2}|\varphi(x)|^{2} \leq c \int_{\Omega}|\nabla \varphi(x)|^{2} d x, \quad c=c\left(c_{1}, K\right)>0 .
$$

D. On va maintenant énoncer quelques conséquences de la propriété (B). On suppose $\Omega$ de type (B) et uniformément régulier. 
Proposition 1.7. Pour $0<r<\frac{1}{2} s \leq 1,2 s \leq t$, on a $G\left(A_{r}, A_{t}\right) \leq$ $c G\left(A_{r}, A_{s}\right) G\left(A_{s}, A_{t}\right)$.

D'après le théorème 1.1 , on a pour $X \in \partial B(0, s)$,

$$
G\left(A_{r}, X\right) \leq c\left\{G\left(A_{r}, A_{s}\right) G\left(A_{s}, X\right)+G\left(A_{r},-A_{s}\right) G\left(-A_{s}, X\right)\right\} .
$$

Cette inégalité s'étend par le principe du maximum à $X \notin B(0, s)$. Faisant $X=A_{t}$ on obtient le résultat voulu grâce à $(\mathrm{PB})$. 口

Corollaire 1.8. Si $0 \in F$ et si $h$ désigne la minimale (normalisée à l'infini) sur $\Omega$, limite de $A_{t}$ pour $t \rightarrow 0, t>0$, on a: $h\left(A_{t}\right) G\left(\infty, A_{t}\right) \sim 1$. $\square$

L'inégalité précédente et la proposition 1.4 donnent pour $r<\frac{1}{2} s, r \leq 1$,

$$
G\left(\infty, A_{r}\right) \sim G\left(\infty, A_{s}\right) G\left(A_{s}, A_{r}\right)
$$

D'où, après division $1 \sim G\left(\infty, A_{s}\right) K_{\left(A_{r}\right)}\left(A_{s}\right), K_{X}$ désignant le noyau Martin normalisé (au point à l'infini). Il ne reste plus qu'à faire tendre $r$ vers 0 . 口

L'énoncé suivant est une variante de la proposition 1.7.

Corollaire 1.9. Soient $X \in \Omega, 0<t \leq 1$, avec $\frac{1}{2} t \leq|X| \leq 2 t, d\left(X, A_{t}\right) \geq$ $c_{0}^{-1} t$, et $G\left(X,-A_{t}\right) \leq c_{0} G\left(X, A_{t}\right)$, pour une constante $c_{0} \geq 1$. On a pour tout $s>0, s \notin\left[\frac{1}{2} t, 2 t\right]$,

(i) $c^{-1} G\left(X, A_{t}\right) G\left(A_{t}, A_{s}\right) \leq G\left(X, A_{s}\right) \leq c G\left(X, A_{t}\right) G\left(A_{t}, A_{s}\right)$,

(ii) si $F$ est symétrique $G\left(-A_{s}, X\right) \leq c G\left(A_{s}, X\right)$, pour chaque $s \leq 1, c=$ $c\left(c_{0}, \Omega\right)>0$.

Preuve. On estime $u(x)=G(X, x)$ à l'aide du théorème 1.1. On obtient

$$
u(x) \leq c u\left(A_{t}\right)\left\{G\left(A_{t}, x\right)+G\left(-A_{t}, x\right)\right\}
$$

pour $x$ tel que $|x|=\frac{1}{2} t$ ou $|x|=2 t$, et donc pour tout $x \in \Omega,|x| \leq \frac{1}{2} t$ ou $|x| \leq 2 t$. D'où, $G\left(X, A_{s}\right) \leq c G\left(X, A_{t}\right) G\left(A_{t}, A_{s}\right)$, et le (i) grace à la proposition 1.4. s'ensuit.

De même, $G\left(X,-A_{s}\right) \leq c G\left(X, A_{t}\right) G\left(-A_{t},-A_{s}\right)$ (d'après (PB)) et le (ii)

Remarque 1.10. Si $X \in \Omega, 0<t \leq 1, \frac{1}{2} t \leq|X| \leq 2 t, d\left(X, A_{t}\right) \geq c_{0}^{-1} t$, et si $G\left(X,-A_{t}\right) \leq c_{0} G\left(X, A_{t}\right)$, il existe $\delta=\delta\left(c_{0}, \Omega\right)>0$ tel que la fonction de Green $g$ de $U=\Omega \backslash \bar{B}(0, t \delta)$ vérifie $g\left(A_{t}, X\right) \geq 2^{-1} G\left(A_{t}, X\right)$ (et a fortiori $\left.g\left(-A_{t}, X\right) \leq 2 c_{0} g\left(A_{t}, X\right)\right)$. En utilisant le théorème 1.1, on voit en effet que

$$
G\left(A_{t}, X\right)-g\left(A_{t}, X\right) \leq c \varepsilon(\delta)\left\{G\left(A_{t}, X\right)+G\left(-A_{t}, X\right)\right\} \leq c\left(1+c_{0}\right) \varepsilon(\delta) G\left(A_{t}, X\right) .
$$




\section{Un critère d'effilement minimal}

On suppose désormais que $0 \in F$, et que $\Omega$ est de type (B) en 0 et uniformément régulier. On note $A_{j}=i 2^{-j}$ et $G$ désigne la fonction de Green de $\Omega$. Pour $u$ surharmonique $>0$ sur $\Omega$, et $A \subset \Omega$, on note $R[u, A]$ la réduite de $u$ sur $A$ (relativement à $\Omega$ ) [Na]. Rappelons qu'une partie $\Phi \subset \Omega$ est $h$-effilée ( $h$ est la minimale sur $\Omega$ associée à $\left.\left\{A_{j}\right\}\right)$ si $R[\Phi, h]$ est un potentiel, ou encore si $R[\Phi, h] \neq h$ (cf. [Na]).

Soient $\left\{x_{j}\right\}$ une suite de points de $\Omega,\left|x_{j}\right| \leq 1, \delta_{j}=\delta\left(x_{j}\right)=d\left(x_{j}, \partial \Omega\right), A_{j}^{\prime}$ le point $A_{k}$ tel que $2^{-k-2} \leq\left|x_{j}\right|<2^{-k-1}$. On suppose que pour un $\left.\left.\varepsilon_{0} \in\right] 0, \frac{1}{2}\right]$, $d\left(x_{j}, x_{k}\right)>2 \varepsilon_{0} \delta\left(x_{j}\right)$, si $j \neq k$ et que $G\left(x_{j}, A_{j}^{\prime}\right) \geq \varepsilon_{0} G\left(x_{j},-A_{j}^{\prime}\right)$, pour chaque $j \geq 1$. Soit $\Phi=\cup\left\{B\left(x_{j}, \varepsilon_{0} \delta_{j}\right) ; j \geq 1\right\}$.

Théorème 2.1. L'ensemble $\Phi$ est $h$-efflé minimal si et seulement si

$$
\sum_{j \geq 1} G\left(A_{j}^{\prime}, x_{j}\right)^{2}<\infty
$$

Preuve. On adapte les arguments de $[\mathrm{A} 3$, Section 7$]$ sur l'extension du critère d'effilement de Beurling [Beu] dans le cadre de la géométrie hyperbolique.

1. On montre d'abord que $\Phi$ est $h$-effilé minimal si et seulement si la série $\sum_{n \geq 1} R\left[h, \Phi_{n}\right](\infty)$ converge, où $\Phi_{n}=\cup\left\{B\left(x_{j}, \varepsilon_{0} \delta_{j}\right) ; 2^{-n-2} \leq\left|x_{j}\right| \leq 2^{-n-1}\right\}$ (Condition de type Wiener). Cette condition est trivialement suffisante. tel que:

a) Pour voir que cette condition est nécessaire, on montre qu'il existe $\theta>0$

$$
R\left[h, \bigcup_{j \geq n m} \Phi_{j}\right]+R\left[h, \bigcup_{j \leq n / m} \Phi_{j}\right] \leq(1-\theta) h
$$

sur $\Phi_{n}$ pour tout $n \geq 1$, dès que $m$ est assez grand (si $\Phi$ est $h$-effilé). En fait, le corollaire 1.8, donne pour le potentiel $\pi=R\left[h, \cup_{j \geq n m} \Phi_{j}\right]$

$$
\pi\left(A_{n}\right) \leq c \pi(\infty) h\left(A_{n}\right)=\varepsilon(n m) h\left(A_{n}\right)
$$

(puisque, pour $X \in \cup_{j \geq n m} \Phi_{j}, G\left(X, A_{n}\right) \leq c G(X, \infty)\left\{G\left(A_{n}, \infty\right)\right\}^{-1}$ d'après 1.4). Donc, pour $n m$ assez grand, et si $g$ est la fonction de Green de $\Omega \backslash B\left(0,2^{-n m+1}\right)$

$$
h(X)-\pi(X) \geq \operatorname{ch}\left(A_{n}\right) g\left(A_{n}, X\right) \geq\left(\frac{1}{2} c\right) h\left(A_{n}\right) G\left(A_{n}, X\right) \geq c^{\prime} h(X),
$$

pour chaque $X \in \Phi_{n}$ d'après la remarque 1.8. De sorte que $\pi \leq\left(1-c^{\prime \prime}\right) h$ sur $\Phi_{n}$ pour un $c^{\prime \prime}>0$, si $m$ est choisi assez grand. D'autre part, toujours sur $\Phi_{n}$,

$$
R\left[h, \bigcup_{j \leq n / m} \Phi_{j}\right] \leq c \max \left\{h(u) ;|u| \geq 2^{-n / m}\right\} G_{A_{n}} \leq c\left\{G\left(A_{n}, A_{[n / m]}\right)\right\}^{-1} h
$$


d'où $(*)$, compte tenu de 1.5 .

b) Soient $\mu=-\Delta\left\{R\left[h, \cup_{n \geq 1} \Phi_{n m}\right]\right\}, \mu_{n}=\mathbf{1}_{\Phi_{n}} \cdot \mu$; d'après $(*), G\left(\mu_{n}\right) \geq \theta h$ sur $\Phi_{n m}$. D'où

$$
\sum_{n \geq 1} R\left[h, \Phi_{n m}\right](\infty) \leq \theta^{-1} \sum_{n \geq 1} G \mu_{n}(\infty) \leq \theta^{-1} h(\infty)<\infty .
$$

On montre de même que $\sum_{n \geq 1} R\left[h, \Phi_{n m+p}\right](\infty)<\infty$ si $1 \leq p<m$.

2. On a, $c_{1}^{-1} R\left[G_{A n}, \Phi_{n}\right]\left(A_{n}\right) \leq R\left[h, \Phi_{n}\right](\infty) \leq c_{1} R\left[G_{A n}, \Phi_{n}\right]\left(A_{n}\right)$ pour tout $n \geq 1$ et une constante $c_{1}>0$ convenable. En effet, d'après 1.8 et 1.9,

$$
h \sim h\left(A_{n}\right) G_{A n} \sim\left\{G\left(A_{n}, \infty\right)\right\}^{-1} G_{A n}
$$

$\operatorname{sur} \Phi_{n}$ et

$$
R\left[G_{A n}, \Phi_{n}\right](\infty) \sim R\left[G_{A n}, \Phi_{n}\right]\left(A_{n}\right) G\left(A_{n}, \infty\right),
$$

d'où $R\left[h, \Phi_{n}\right](\infty) \sim\left\{G\left(A_{n}, \infty\right)\right\}^{-1} R\left[G_{A n}, \Phi_{n}\right](\infty) \sim R\left[G_{A n}, \Phi_{n}\right]\left(A_{n}\right)$. On voit donc que l'effilement minimal de $\Phi$ équivaut à $\sum_{n} R\left[G_{A n}, \Phi_{n}\right]\left(A_{n}\right)<\infty$.

3. On utilise enfin l'identité, pour $\pi=R\left[G_{A n}, \Phi_{n}\right], \pi\left(A_{n}\right)=\int_{\Omega}|\nabla \pi|^{2} d x$ qu'on combine avec l'inégalité $\int_{\delta \leq 1}|\varphi(x)|^{2} \delta^{-2}(x) d x \leq c \int_{\Omega}|\nabla \varphi|^{2} d x$, pour chaque $\varphi \in H_{0}^{1}(\Omega)$ (cf.Section 1). D'où:

$$
\pi\left(A_{n}\right) \geq c \int_{\Phi_{n}}|\pi|^{2} \delta^{-2} d x=c \int_{\Phi_{n}}\left|G_{A n}\right|^{2} \delta^{-2} d x \geq c^{\prime} \sum_{j \in J_{n}}\left\{G\left(A_{n}, x_{j}\right)\right\}^{2},
$$

$J_{n}=\left\{j ; A_{j}^{\prime}=A_{n}\right\}$.

L'estimée opposée est immédiate puisque $\pi \leq \sum_{j \in J_{n}} R\left[G_{A n}, B_{j}\right]$ et que d'après Harnack et la définition de la réduite

$$
R\left[G_{A n}, B_{j}\right]\left(A_{n}\right) \leq c G\left(A_{n}, x_{j}\right) G\left(x_{j}, A_{n}\right) \text {. 口 }
$$

Corollaire 2.2. Soit $\Phi^{\prime}$ la réunion d'une collection $\left\{I_{j}\right\}_{j \geq 1}$ de composantes de $\Gamma \cap \Omega$ et soit $x_{j} \in I_{j}$ le "milieu" de $I_{j}$. Supposons que $G\left(x_{j}, A_{j}^{\prime}\right) \geq$ $\varepsilon_{0} G\left(x_{j},-A_{j}^{\prime}\right)$ et $\delta_{j}=\delta\left(x_{j}\right) \leq \frac{1}{2}\left|x_{j}\right|$. Alors, $\Phi^{\prime}$ est $h$-efflée si et seulement si $\sum_{j \geq 1} G\left(x_{j}, A_{j}^{\prime}\right)^{2}<\infty$.

Il faut observer ici que d'après le théorème 1.1 , si $s$ surharmonique sur $\Omega$ majore $h$ sur $B\left(x_{j}, 2^{-1} \delta_{j}\right)$ alors $s \geq c h$ sur $I_{j}$, avec $c=c(K)>0$.

Remarques. 1. On n'utilisera au paragraphe 4 le théorème 2.1 que pour le cas où $J_{n}$ est réduit à un élément; l'utilisation de l'énergie dans la troisième partie de la démonstration est alors inutile, et l'équivalence $\pi\left(A_{n}\right) \sim G\left(A_{n}, x_{j}\right)^{2}$ immédiate.

2. Lorsque $F \subset \mathbf{R}$ (ou même si $\Gamma$ est $C^{2}$ ), 0 est un point Martin double si et seulement si $\Gamma \cap \Omega$ est $h$-effilé. Il découle alors du théorème précédent que 0 est double si et seulement si $\sum_{n \geq 1} \sum_{j \in J_{n}} G\left(A_{n}, x_{j}\right)^{2}<\infty$, si $\left\{x_{j}\right\}$ est la suite des centres des intervalles contigus de $F$ (on suppose toujours $\Omega$ uniformément régulier). On obtient ainsi un critère équivalent à celui de Benedicks ([B]). 


\section{Un critère de multiplicité}

Dans cette partie on suppose $\Omega$ symétrique par rapport à 0 (voir le 3.2) et uniformément régulier. Soient $\Gamma_{1}$ le graphe d'une fonction $f_{1}: \mathbf{R} \rightarrow \mathbf{R}$ lipschitzienne, impaire telle que $\Gamma_{1} \supset F$, et $g_{j}$ la fonction de Green de $\omega_{j}=\{\Omega \backslash \Gamma\} \cup\{x \notin$ $\left.F ; a^{-1} 2^{-j}<|x|<a 2^{-j+1}\right\}$ (a constante $>1$ fixée). On pose $A_{j}=i 2^{-j}$ et $B_{j}=-A_{j}$ pour $j \geq 1$.

Théorème 3.1. Le point 0 est un point Martin double pour $\Omega$ si et seulement si $\sum_{j \geq 1} g_{j}\left(A_{j}, B_{j}\right)<\infty$.

Preuve. A: La condition est suffisante. Pour $j, k \geq 1, j \neq k, G\left(A_{k}, X\right) \leq$ $c\left\{G\left(A_{k}, A_{j}\right) g_{j}\left(A_{j}, X\right)+G\left(A_{k}, B_{j}\right) g_{j}\left(B_{j}, X\right)\right\}$ si $X \in \Gamma_{1} \cap \Omega, 2^{-j} \leq|X| \leq 2^{-j+1}$ (grâce au Théorème 1.1). D'où aussi, d'après (PB),

$$
G\left(A_{k}, X\right) \leq c G\left(A_{k}, A_{j}\right)\left\{g_{j}\left(A_{j}, X\right)+g_{j}\left(B_{j}, X\right)\right\} .
$$

Notant $u_{k, j}$ la fonction harmonique bornée sur $\Omega_{-}=\left\{x+i y ; y<f_{1}(x)\right\}$ telle que $u_{k, j}(X)=G\left(A_{k}, X\right)$ si $X \in \Gamma_{1}, 2^{-j} \leq|X| \leq 2^{-j+1}$ et $u_{k, j}(X)=0$ si $X \in \Gamma_{1} \backslash\left\{X ; 2^{-j} \leq|X| \leq 2^{-j+1}\right\}$,

$$
u_{k, j}(X) \leq c G\left(A_{k}, A_{j}\right)\left\{g_{j}\left(A_{j}, X\right)+g_{j}\left(B_{j},-X\right)\right\}=2 c G\left(A_{k}, A_{j}\right) g_{j}\left(A_{j}, X\right)
$$

pour $X \in \Omega$. D'où, en utilisant le théorème 1.1 , remarque 1.2 , si $m \neq j$,

$$
u_{k, j}\left(B_{m}\right) \leq 2 c G\left(A_{k}, A_{j}\right) g_{j}\left(A_{j}, B_{j}\right) G\left(B_{j}, B_{m}\right) .
$$

On peut alors estimer $G\left(A_{k}, B_{m}\right), k>m$, à l'aide des quantités $\gamma_{j}=$ $g_{j}\left(A_{j}, B_{j}\right)$. Pour $s<m<k$, on a (en convenant de remplacer $G\left(A_{k}, A_{k}\right)$ et $G\left(B_{m}, B_{m}\right)$ par 1$)$

$$
\begin{aligned}
G\left(A_{k}, B_{m}\right) & \leq c^{\prime} \sum_{j \geq 1} \gamma_{j} G\left(A_{k}, A_{j}\right) G\left(B_{j}, B_{m}\right)+c^{\prime} G\left(A_{k}, A_{1}\right) G\left(B_{1}, B_{m}\right) \\
& \leq c^{\prime \prime} G\left(A_{k}, A_{s}\right)\left\{\sum_{j<s} \gamma_{j}\right\}+c^{\prime \prime} G\left(A_{k}, A_{m}\right)\left\{\sum_{j \geq s} \gamma_{j}\right\}+c^{\prime \prime} G\left(A_{k}, A_{1}\right)
\end{aligned}
$$

(compte tenu des inégalités $G\left(B_{j}, B_{k}\right)=G\left(A_{j}, A_{k}\right) \leq c$ pour $k \neq n, G\left(A_{k}, A_{j}\right) \leq$ $c G\left(A_{k}, A_{s}\right)$ pour $k<s<j$, et $\left.G\left(A_{k}, A_{j}\right) G\left(A_{j}, A_{m}\right) \leq c G\left(A_{k}, A_{m}\right)\right)$. La minimale $h$ sur $\Omega$ attachée à l'accès par le haut en 0 est donc telle que

$$
h\left(B_{m}\right) \leq \operatorname{ch}\left(A_{s}\right)+\operatorname{ch}\left(A_{m}\right)\left\{\sum_{j \geq s} \gamma_{j}\right\}+c^{\prime \prime} h\left(A_{1}\right)
$$

et

$$
h\left(B_{m}\right) \leq \operatorname{ch}\left(A_{m}\right)\left\{\sum_{j \geq s} \gamma_{j}+G\left(A_{m}, A_{s}\right)+G\left(A_{m}, A_{1}\right)\right\} .
$$

D'où, $\lim _{m \rightarrow \infty} h\left(B_{m}\right) / h\left(A_{m}\right)=0$, et 0 est bien un point double. $\square$ 
B: La condition est nécessaire. Supposons que 0 soit double pour $\Omega$. Notons $h$ (respectivement $k$ ) la minimale sur associée à la suite $\left\{A_{n}\right\}$ (respectivement $\left.\left\{B_{n}\right\}\right)$ et introduisons $T=\{x+i y ; y>2 K|x| ;|x+i y| \leq 1\}, K$ désignant la constante de lipschitz de $f_{1}$. a) Il est clair que $T$ est $k$-effilé (puisque $k$ n'est pas adhérent à $T$ dans le compactifié de Martin). De plus, si $v_{j}=G\left(B_{j}, \cdot\right) / G\left(B_{j}, \infty\right)$, $\lim \sup _{k \rightarrow \infty} R\left[v_{j} ; T\right](\infty)<1$; en effet, sur le bord d'une boule $B\left(B_{j}, c 2^{-j}\right)(c$ constante $>0$ petite fixée) on a $v_{j} \sim k\left(B_{j}\right)$ (Corollaire 1.8). Donc $v_{j} \leq c k$ hors de $B\left(B_{j}, c 2^{-j}\right)$ et en particulier sur $T$. On en déduit, par convergence dominée, que $\lim \sup _{j \rightarrow \infty} R\left[v_{j} ; T\right](\infty)=R[k ; T](\infty)<1$ (d'après l'effilement de $T$ en $k$ ).

b) On voit alors que si $g_{T}$ désigne la fonction de Green de $U=\Omega \backslash T$, on a $\liminf _{j \rightarrow \infty} g_{T}\left(B_{j}, \infty\right) / G\left(B_{j}, \infty\right)>0$. Il suffit d'observer que

$g_{T}\left(B_{j}, \infty\right) / G\left(B_{j}, \infty\right)=\left\{G\left(B_{j}, \infty\right)-R\left[G_{B_{j}}, T\right](\infty)\right\} / G\left(B_{j}, \infty\right)=1-R\left[v_{j}, T\right](\infty)$.

D'après la propriété de Harnack, on a donc aussi $g_{T}\left(B_{j}, B_{1}\right) \geq c G\left(B_{j}, B_{1}\right)$, pour chaque $j \geq 2$, pour une constante $c>0$ (qui dépend de $\Omega$ ).

c) Minorons maintenant $G\left(A_{j}, B_{1}\right)$ en écrivant d'abord $G_{A_{j}}$ comme solution d'un problème de Dirichlet sur $U$. On a $G\left(A_{j}, B_{1}\right)=\sum_{k \geq 0} w_{j, k}\left(B_{1}\right)$ pour $w_{j, k}$ harmonique sur $U$ avec $w_{j, k}=G_{A_{j}}$ sur $\partial T \cap\left\{z ; 2^{-k-1}<|z| \leq 2^{-k}\right\}$; d'où sans aucune difficulté (en utilisant le principe du maximum, puis le point précédent de la démonstration)

$$
G\left(A_{j}, B_{1}\right) \geq c \sum_{1<k<j} G\left(A_{j}, A_{k}\right) \gamma_{k} g_{T}\left(B_{k}, B_{1}\right) \geq c^{\prime} \sum_{1<k<j} G\left(A_{j}, A_{k}\right) \gamma_{k} G\left(B_{k}, B_{1}\right)
$$

si $\gamma_{k}=g_{k}\left(A_{k}, B_{k}\right)$. Enfin, d'après l'égalité $G\left(A_{j}, A_{k}\right)=G\left(B_{k}, B_{1}\right)$ et la proposition 1.7,

$$
G\left(A_{j}, B_{1}\right) \geq c^{\prime \prime} G\left(A_{j}, A_{1}\right) \sum_{1<k<j} g_{k}\left(A_{k}, B_{k}\right) \geq c^{\prime \prime \prime} G\left(A_{j}, B_{1}\right) \sum_{1<k<j} g_{k}\left(A_{k}, B_{k}\right) .
$$

Ce qui achève bien entendu la preuve du théorème. $\square$

Remarque 3.2. Comme le montrent de simples modifications de la preuve précédente, le théorème 3.1 s'étend au cas où $F=\hat{\mathbf{C}} \backslash \Omega$ est contenu dans le graphe d'une fonction $C^{1, \alpha},(\alpha>0), \Omega$ n'étant plus supposé symétrique.

\section{Construction d'un exemple}

Soit $\Gamma$ la courbe plane "lipschitzienne" obtenue de la manière suivante. Si $\sigma$ désigne la réunion des deux segments $\left[1,1+(1 / \sqrt{2}) e^{i \pi / 4}\right],\left[1+(1 / \sqrt{2}) e^{i \pi / 4}, 2\right], \Gamma$ est la réunion de ses homothétiques $\pm 2^{-j} \sigma, j \geq 1$, augmentée de l'origine 0 .

On notera $C_{k}=2^{-k}\left(1+(1 / \sqrt{2}) e^{i \pi / 4}\right), k \geq 1$, les "pointes" tournées vers le haut de $\Gamma, D_{k}=-C_{k}$ les pointes symétriques, $A_{n}=i 2^{-n}$, et $B_{n}=-A_{n}$. $\Omega_{-}$ désignera la région délimitée par $\Gamma_{1}=\Gamma \cup\{z \in \mathbf{R} ;|z| \geq 1\}$ et contenant $-i$. 
Si $\varepsilon=\left\{\varepsilon_{j}\right\}_{j \geq 1}$ est une suite de réels $\geq 0$ tels que $\varepsilon_{j} \geq 10^{-1}$, on notera $\Omega(\varepsilon)$ le domaine $\hat{\mathbf{C}} \backslash \Gamma$ augmenté de tous les "trous" $\Gamma \cap B\left(C_{k}, \varepsilon_{k} 2^{-k}\right)$ et $\Gamma \cap B\left(D_{k}, \varepsilon_{k} 2^{-k}\right)$, $k \geq 1 ; \check{\Omega}(\varepsilon)$ désignera la région obtenue en rajoutant à $\hat{\mathbf{C}} \backslash \Gamma$ les "trous" à gauche $\Gamma \cap B\left(D_{k}, \varepsilon_{k} 2^{-k}\right), k \geq 1$.

Il est clair que les domaines $\Omega(\varepsilon)$ et $\check{\Omega}(\varepsilon)$ sont uniformément réguliers et qu'il vérifient (UR) avec une constante $c_{1}$ indépendante de $\varepsilon$.

Notre construction sera basée sur les théorèmes 2.1 et 3.1 , combinés avec l'estimée élémentaire et standard suivante.

Lemme 4.1. Soient $\varepsilon>0, \varepsilon<10^{-1}$, et $\omega_{\varepsilon}=\left\{r e^{i \varphi} ; r<1, \varphi \neq-\pi / 4, \varphi \neq\right.$ $5 \pi / 4 \bmod (2 \pi)\} \cup B(0, \varepsilon)$. Notons $A=\frac{1}{2} i$ et $B=-\frac{1}{2} i$. La fonction de Green $G_{\varepsilon}$ de $\omega_{\varepsilon}$ vérifie pour $\varepsilon \rightarrow 0$,

$$
G_{\varepsilon}(A, 0) \sim \varepsilon^{2 / 3}, \quad G_{\varepsilon}(B, 0) \sim \varepsilon^{2} \quad \text { et } \quad G_{\varepsilon}(A, B) \sim \varepsilon^{8 / 3} .
$$

Remarque 4.2. Soit, avec les notations précédentes, $u$ (respectivement $v$ ) la mesure harmonique dans $\omega_{\varepsilon}$ de $\left\{e^{i \alpha} ;-\pi / 4<\alpha<5 \pi / 4\right\}$ (respectivement $\left.\left\{e^{i \alpha} ; 5 \pi / 4<\alpha<7 \pi / 4\right\}\right) . u(X)$ et $G_{\varepsilon}(A, X)$ ont même ordre de grandeur (pour $\varepsilon \rightarrow 0)$ au point $X=\frac{1}{2} A$ et donc aussi d'après le principe de Harnack au bord, pour $|X|=\frac{1}{4}$, et $-\pi / 4<\arg (X)<5 \pi / 4$. D'où, avec le principe du maximum: $u(0) \sim G_{\varepsilon}(A, 0)$; de même $v(0) \sim G_{\varepsilon}(B, 0)$. En particulier $v(0)$ est infiniment petit devant $u(0)$ pour $\varepsilon \rightarrow 0<4$.

Corollaire 4.3. Soient $j$ un entier $\geq 1, \varepsilon_{j}>0$, et $g_{j}$ la fonction de Green du domaine $\Omega=\left(\mathbf{C} \backslash \Gamma_{1}\right) \cup B\left(C_{j}, 2^{-j} \varepsilon_{j}\right), \Gamma_{1}=\Gamma \cup\{z \in \mathbf{R} ;|z| \geq 1\}$. On a, avec une constante absolue $C \geq 1, C^{-1} \varepsilon_{j}^{2 / 3} \leq g_{j}\left(A_{j}, C_{j}\right) \leq C \varepsilon_{j}^{2 / 3}, C^{-1} \varepsilon_{j}^{2} \leq g_{j}\left(B_{j}, C_{j}\right) \leq$ $C \varepsilon_{j}^{2}$ et

$$
C^{-1} \varepsilon_{j}^{8 / 3} \leq g_{j}\left(A_{j}, B_{j}\right) \leq C \varepsilon_{j}^{8 / 3} .
$$

La fonction de Green du domaine symétrique $\Omega^{\prime}=\Omega \cup B\left(D_{j}, 2^{-j} \varepsilon_{j}\right)$, et celle de $\Omega^{\prime \prime}=\Omega^{\prime} \cap\left\{z ; 2^{-j-1}<|z|<2^{-j+1}\right\}$ vérifient des inégalités analogues. Notons les conséquences suivantes pour la fonction de Green $G$ d'un domaine $\Omega(\varepsilon)$ général.

Lemme 4.4. Pour $k$, $j$ entiers $\geq 1$, on a: (i) $G\left(A_{j}, D_{k}\right)=G\left(B_{j}, C_{k}\right) \leq$ $c G\left(A_{j}, C_{k}\right)$, (ii) si $j \neq k, G\left(A_{j}, C_{k}\right) \sim G\left(A_{j}, A_{k}\right) G\left(A_{k}, C_{k}\right)$ (équivalence uniforme relativement à $\left\{\varepsilon_{j}\right\}, c>0$ indépendant de $\left.\left\{\varepsilon_{j}\right\}\right)$.

Preuve. Observons d'abord que $G\left(C_{k}, B_{k}\right) \leq c G\left(C_{k}, A_{k}\right) . G\left(B_{k}, C_{k}\right)$ est, en effet, majoré par (un multiple de) la mesure harmonique, au point $C_{k}$, de $\partial B\left(C_{k}, 2^{-k-1}\right)$ dans $\omega_{k}=B\left(C_{k}, 2^{-k-1}\right) \backslash\left\{\Gamma \backslash B\left(C_{k}, \varepsilon_{k} 2^{-k}\right)\right\}$ alors que $G\left(C_{k}, A_{k}\right)$ est minoré par (une constante fois) la mesure harmonique de $\partial B\left(C_{k}, 2^{-k-1}\right) \cap \Omega$ dans $\omega_{k}$, au point $C_{k}$. D'où l'assertion, par la remarque 4.2. Il ne reste plus qu'à appliquer le corollaire 1.9. $\square$ 
L'assertion suivante résout les questions de $[\mathrm{Ch}]$ rappelées au début.

Théorème 4.5. Il existe une suite $\left\{\varepsilon_{j}\right\}_{j \geq 1}, 0 \leq \varepsilon_{j}<1 / 10$, telle que l'origine soit d'une part un point Martin double pour $\Omega(\varepsilon)$, et, d'autre part, un point Martin simple pour $\check{\Omega}(\varepsilon)$. De plus, relativement au domaine $\Omega=\Omega(\varepsilon)$ et à chacune des 2 minimales sur $\Omega$ associées à 0 , la partie $\Gamma \cap \Omega$ du graphe $\Gamma$ contenue dans $\Omega$ est non effilée au sens minimal.

Remarquons d'abord qu'on déduit aisément des théorème 2.1 et 3.1 l'existence une suite $\varepsilon=\left\{\varepsilon_{j}\right\}_{j \geq 1}, 0 \leq \varepsilon_{j}<10^{-1}$ telle que 0 soit relativement à $\Omega=\Omega(\varepsilon)$ un point Martin double, et qu'en même temps $\sum_{d}=\Omega \cap \Gamma \cap\{z ; \operatorname{Re}(z)>0\}$ ne soit pas $h$-effilée (si $h$ est la minimale sur $\Omega(\varepsilon)$ associée à $\left\{A_{j}\right\}_{j \geq 1}$ ). Il suffit de prendre $\varepsilon_{j}=10^{-1} j^{-3 / 4}$, les propriétés voulues résultant alors de $4.3,2.1$, et 3.1.

Le choix d'une suite $\left\{\varepsilon_{j}\right\}$ telle que l'origine soit de plus un point Martin simple pour $\check{\Omega}(\varepsilon)$ est plus délicat; il faudra recourir à une suite $\left\{\varepsilon_{j}\right\}$ plus irrégulière.

Fixons une fois pour toute $\varepsilon_{j}=(1 / 10) j^{-3 / 4}$ pour $j$ impair et soit $\left\{k_{m}\right\}$ une suite strictement croissante d'entiers pairs (à choisir ultérieurement). Notons $\Omega=\Omega(\varepsilon)$ le domaine associé à la suite $\varepsilon$ définie par $\varepsilon_{k_{m}}=(1 / 10) m^{-3 / 4}$ pour $m \geq 1$, et $\varepsilon_{j}=0$ pour $j$ pair, $j \notin\left\{k_{m} ; m \geq 1\right\}$. On va voir que si la suite $\left\{k_{j}\right\}$ est très rapidement croissante, $\Omega$ vérifie les assertions du théorème 4.5 .

Notons

$$
\Omega_{0}=(\hat{\mathbf{C}} \backslash \Gamma) \cup\left(\cup_{k \geq 0} B\left(C_{2 k+1}, \varepsilon_{2 k+1} 2^{-2 k-1}\right)\right) \cup\left(\cup_{k \geq 0} B\left(D_{2 k+1}, \varepsilon_{2 k+1} 2^{-2 k-1}\right)\right),
$$

et $\Omega_{j}$ le domaine symétrique

$$
\Omega_{j}=\Omega_{0} \cup\left\{\cup_{m \leq j} B\left(C_{k_{m}}, 2^{-k_{m}} \varepsilon_{m}\right)\right\} \cup\left\{\cup_{m \leq j} B\left(D_{k_{m}}, 2^{-k_{m}} \varepsilon_{m}\right)\right\}
$$

et $\check{\Omega}_{j}$ le domaine "tordu" $\Omega_{0} \cup\left\{\cup_{m \leq j} B\left(D_{k_{m}}, 2^{-k_{m}} \varepsilon_{m}\right)\right\}$; ces domaines sont définis dès que les $k_{m}, m \leq j$, ont été choisis. On notera $G_{j}$ (respectivement $\bar{G}_{j}$ ) la fonction de Green de $\Omega_{j}$ (respectivement $\check{\Omega}_{j}$ ) et on s'appuiera sur les lemmes suivants.

Lemme 4.6. L'entier $j$ étant fixé, la fonction de Green $q_{j}=\bar{G}_{j}(\infty, \cdot)$ de pôle le point à l'infini dans $\check{\Omega}_{j}$ vérifie $\lim _{t>0, t \rightarrow 0}\left\{q_{j}\left(x_{t}\right) / q_{j}\left(-x_{t}\right)\right\}=0$, où $x_{t}=i t$.

Raisonnons pour $j=0$. Si $x=x_{t}, t>0$ petit, et $q=q_{0}$, on a:

$$
q(x) / q(-x)=\left\{1-R\left[K_{x}, \sum_{d}\right](\infty)\right\} /\left\{1-R\left[K_{-x}, \sum_{d}\right](\infty)\right\}
$$

où $K_{x}(y)=G(x, y) / G(x, \infty)$ est le noyau Martin de $\Omega_{0}=\Omega(\varepsilon)$ (normalisé à l'infini), $G$ la fonction de Green de $\Omega_{0}$. Notons $h=\lim _{t \rightarrow 0} K_{x}, k=\lim _{t \rightarrow 0} K_{-x}$. D'après le choix des $\varepsilon_{2 j+1}, h \neq k$ (Théorème 3.1). 
Comme pour $x \in \sum_{d}, G_{x}\left(B_{j}\right) \leq c G_{x}\left(A_{j}\right)$, toute minimale $u$ sur $\Omega$ adhérente à $\sum_{d}$, vérifie $u\left(B_{j}\right) \leq c u\left(A_{j}\right)$ et $k$ ne peut donc pas adhérer à $\sum_{d}$ dans le compactifié de Martin de $\Omega$. A fortiori $\sum_{d}$ est $k$-effilé, et $R\left[k, \sum_{d}\right](\infty)<1$.

On vérifie ensuite que:

$$
\lim _{t \rightarrow 0} 1-R\left[K_{-x}, \sum_{d}\right](\infty)=1-R\left[k, \sum_{d}\right](\infty)>0
$$

en utilisant le théorème de convergence dominée exactement comme dans la preuve du théorème 3.1 (partie B.a)). Comme d'autre part (d'après le lemme de Fatou) $\lim _{t \rightarrow 0} 1-R\left[K_{x}, \sum_{d}\right](\infty)=0$, le résultat voulu découle alors de (4.2).

Dans l'énoncé suivant, $g_{n}$ désigne la fonction de Green du domaine $\omega=$ $\left\{\hat{\mathbf{C}} \backslash \Gamma_{1}\right\} \cup B\left(D_{k_{n}}, 2^{-k_{n}} \varepsilon_{k_{n}}\right), \Gamma_{1}=\Gamma_{0} \cup\{z \in \mathbf{R} ;|z| \geq 1\}$.

Lemme 4.7. Supposons choisis $k_{1}, k_{2}, \ldots, k_{n-1}$. On a

(1) $\lim _{m \rightarrow \infty} \bar{G}_{n-1}\left(A_{m}, B_{m}\right)=0$,

(2) $\lim _{k_{n} \rightarrow \infty} \bar{G}_{n}\left(A_{k_{n}}, B_{k_{n}}\right) / g_{n}\left(A_{k_{n}}, B_{k_{n}}\right)=1$,

(3) $\lim _{k_{n} \rightarrow \infty} \bar{G}_{n}\left(A_{k_{n}}, D_{k_{n}}\right) / g_{n}\left(A_{k_{n}}, D_{k_{n}}\right)=1$.

Preuve. (1) découle du lemme précédent, puisque par le principe du maximum $\bar{G}_{n-1}\left(A_{m}, \infty\right) \geq c G_{n-1}\left(A_{m}, B_{m}\right) \times \bar{G}_{n-1}\left(B_{m}, \infty\right)$.

Pour le (2), on écrit d'abord que $\bar{G}_{n}\left(B_{k_{n}}, A_{k_{n}}\right)=g_{n}\left(B_{k_{n}}, A_{k_{n}}\right)+w\left(B_{k_{n}}\right), w$ désignant la réduite (relativement à $\check{\Omega}_{n}$ ) de $u=\bar{G}_{n}\left(A_{k_{n}}, \cdot\right)$ sur $\Gamma_{1} \cap \breve{\Omega}_{n-1}$.

Comme $u$ est majorée par une constante absolue hors de $B\left(A_{k_{n}}, 2^{-k_{n}}\right)$, et comme la taille relative des "trous" de $\check{\Omega}_{n-1} \cap \Omega_{0}$ (les $B\left(D_{m}, 2^{-m} \varepsilon_{m}\right) \cap \Gamma, m$ impair, ou $\left.m=k_{j}, j<n\right)$ tend vers 0 pour $m \rightarrow \infty$, on a, uniformément par rapport à $k_{n}, \lim _{m \rightarrow \infty} u\left(D_{2 m+1}\right)=0 . u(x)$ tend donc vers 0 si $x$ tend vers 0 le long de $\Gamma_{0} \cap \check{\Omega}_{n-1}$ (uniformément par rapport à $k_{n}$ ). On en déduit que $\lim _{k_{n} \rightarrow \infty} w\left(B_{k_{n}}\right)=0$; le (2) s'ensuit puisque $g_{n}\left(A_{k_{n}}, B_{k_{n}}\right)$ est de l'ordre d'une constante à $n$ fixé. Le (3) s'établit de même. $\square$

Lemme 4.8. Si $C$ est une constante $>0$ assez grande, on peut déterminer de proche en proche des entiers pairs $k_{1}<k_{2}<\cdots<k_{j}<\cdots$ tels que pour chaque $j \geq 1$ et $m \leq j$

$$
q_{j}\left(A_{k_{m}}\right)<C m^{-2} q_{j}\left(B_{k_{m}}\right) .
$$

Comme $\varepsilon_{k j}=10^{-1} j^{-3 / 4}$, on a $g_{j}\left(A_{k j}, B_{k j}\right) \sim j^{-2}$, uniformemént par rapport au choix de $k_{j}$. Rappelons qu'on note $g_{j}$ la fonction de Green de $\omega_{j}=\left(\hat{\mathbf{C}} \backslash \Gamma_{1}\right) \cup$ $B\left(D_{k j} ; 2^{-k j} \varepsilon_{k j}\right), q_{j}=\bar{G}_{k}(\infty, \cdot)$ celle de $\check{\Omega}_{k}$ avec pôle le point à l'infini.

Supposons que $k_{1}, k_{2}, \ldots, k_{n-1}$ aient été déterminés de telle sorte que les relations (1) soient vérifiées pour $m \leq j<n$ et un certain $C=C_{1}$. Il est d'abord clair que si $k_{n}$ est choisi assez grand, (1) sera encore vérifiée pour $j=n$ et $m<n$ (puisque $q_{n} \rightarrow q_{n-1}$ simplement lorsque $k_{n} \rightarrow \infty$ ).

Le problème est donc de voir si pour $k_{n}$ assez grand on a:

$$
q_{n}\left(A_{k_{n}}\right) \leq C n^{-2} q_{n}\left(B_{k_{n}}\right) .
$$


Décomposons $q_{n}$ en $q_{n}=q_{n-1}+\varphi$ où $\varphi$ est la fonction harmonique bornée sur $\Omega_{n-1}$ admettant pour valeurs au bord $q_{n}\left(q_{n}\right.$ sur $\Gamma \cap B\left(D_{k_{n}}, 2^{-k_{n}} \varepsilon_{k_{n}}\right)$ et 0 ailleurs). D'après le lemme 4.6 , on a, pour $\varepsilon>0$ donné,

$$
q_{n-1}\left(A_{k_{n}}\right) \leq \varepsilon^{n-2} q_{n-1}\left(B_{k_{n}}\right)
$$

si $k_{n}$ est assez grand. D'autre part, par le principe du maximum

$$
\varphi\left(A_{k_{n}}\right) \leq c q_{n}\left(D_{k_{n}}\right) \times \bar{G}_{n}\left(D_{k_{n}}, A_{k_{n}}\right) .
$$

On a donc d'après les lemmes $4.4,4.6$ et le théorème 1.2, pour $k_{n}$ assez grand, (et une nouvelle constante $c$ indépendante de $n$ ),

$$
\begin{aligned}
\varphi\left(A_{k_{n}}\right) & \leq c q_{n}\left(D_{k_{n}}\right) \times \bar{G}_{n}\left(D_{k_{n}}, A_{k_{n}}\right) \\
& \leq c q_{n}\left(B_{k_{n}}\right) \bar{G}_{n}\left(B_{k_{n}}, D_{k_{n}}\right) \bar{G}_{n}\left(D_{k_{n}}, A_{k_{n}}\right) .
\end{aligned}
$$

Mais d'après le principe du maximum (Proposition 1.4), et le lemme 4.7 (2)

$$
\bar{G}_{n}\left(B_{k_{n}}, D_{k_{n}}\right) \bar{G}_{n}\left(D_{k_{n}}, A_{k_{n}}\right) \leq c \bar{G}_{n}\left(B_{k_{n}}, A_{k_{n}}\right) \leq C_{2} n^{-2}
$$

où $C_{2}$ est une constante indépendante de $n$ (et de $C_{1}$ ).

Finalement, si $C_{1}>C_{2}$, on a pour $k_{n}$ assez grand: $q_{n}\left(A_{k_{n}}\right)<C_{1} n^{-2} q_{n}\left(B_{k_{n}}\right)$.

Concluons:

Proposition 4.9. Les $k_{n}$ étant successivement choisis comme dans le lemme 4.8 , le domaine $\breve{\Omega}_{\infty}$ ne présente qu'une minimale associée à 0 , alors que pour le domaine $\Omega_{\infty}$ il existe deux minimales indépendantes associées à 0 .

L'assertion pour le domaine $\Omega-\infty$ découle du théorème 3.1 , et du choix qui a été fait de la taille des "trous" autour des $C_{j}$ et $D_{j}$.

D'autre part notant $\bar{G}$ la fonction de Green de $\check{\Omega}_{\infty}=\cup_{j \geq 1} \check{\Omega}_{j}$, et $q=\bar{G}(\infty, \cdot)$, on a d'après le choix des $k_{j}$

$$
q\left(A_{k_{j}}\right) \leq c q\left(B_{k j}\right) \bar{G}\left(A_{k j}, B_{k j}\right) .
$$

Or (Proposition 1.4), $\bar{G}\left(A_{k_{j+m}}, B_{k_{j}}\right) \geq c \bar{G}\left(A_{k_{j+m}}, B_{k_{j+m}}\right) \times \bar{G}\left(B_{k_{j+m}}, B_{k_{j}}\right)$. On en déduit, $\bar{G}\left(A_{k_{j+m}}, B_{k_{j}}\right) \geq c \bar{G}\left(B_{k_{j+m}}, B_{k_{j}}\right)\left\{q\left(A_{k_{j+m}}\right) / q\left(B_{k_{j+m}}\right)\right\}$ ou en termes de noyaux-Martin (relatif à $\check{\Omega}_{\infty}$ ), si $K(X, \cdot)=\bar{G}(X, \cdot) / \bar{G}(X, \infty)$,

$$
K\left(A_{k_{j+m}}, B_{k_{j}}\right) \geq c K\left(B_{k_{j+m}}, B_{k_{j}}\right) .
$$

Faisant tendre $m$ vers l'infini, et notant $h$ (respectivement $k$ ) la minimale sur $\check{\Omega}_{\infty}$ attachée à la suite $\left\{A_{n}\right\}$ (respectivement $\left\{B_{n}\right\}$ ), on a: $h\left(B_{k_{j}}\right) \geq c k\left(B_{k_{j}}\right)$. Ce qui entraine que $h=k$ et que 0 est un point simple. $\square$ 


\section{Références}

[A1] Ancona, A.: Une propriété de la compactification de Martin d'un domaine euclidien. Ann. Inst. Fourier (Grenoble) 29, 1979, 71-90.

[A2] Ancona, A.: Régularité d'accès des bouts et frontière de Martin d'un domaine euclidien. - J. Math. Pures et Appl. 63, 1984, 215-260.

[A3] Ancona, A.: Positive harmonic functions and hyperbolicity. - Potential Theory, Surveys and Problems, Prague, 1987. Lecture Notes in Mathematics 1344. Springer-Verlag, Berlin-Heidelberg, 1988.

[A4] Ancona, A.: On strong barriers and an inequality of Hardy for domains in $\mathbf{R}^{n}$. - J. London Math. Soc. (2) 34, 1986, 274-290.

[B] Benedicks, M.: Positive harmonic functions vanishing on the boundary of certain domains in $\mathbf{R}^{n}$. - Ark. Math. 18, 1980, 53-72.

[Beu] Beurling, A.: A minimum principle for positive harmonic functions. - Ann. Acad. Sci. Fenn. Ser A I Math. 372, 1965, 3-7.

[Ca] Carleson, L.: On $H^{\infty}$ in multiply connected domains. - Conference in honor of A. Zygmund, volume II. Wadworth, 1983, 349-372.

[Ch] Chevallier, N.: Frontière de Martin d'un domaine de $\mathbf{R}^{n}$ dont le bord est inclus dans une hypersurface lipschitzienne. - Ark. Math. 27:1, 1989, 29-48.

[G-J] Garnett, J.B., and P. Jones: The corona theorem for Denjoy domains. - Acta Math. $155,1985,27-40$.

[M] Martin, R.S.: Minimal positive harmonic functions. - Trans. Amer. Math. Soc. 49, 1941, 137-172.

[Na] NaIm, L.: Sur le rôle de la frontière de Martin en théorie du potentiel. - Ann. Inst. Fourier (Grenoble) 7, 1957, 183-281.

[Z] Zinsmeister, M.: Espaces de Hardy et domaines de Denjoy. - Ark. Math. (à paraitre).

Université de Paris 11

Bâtiment 425

Campus d'Orsay

F-91405 Orsay Cedex

France

Received 3 July 1989 